\title{
Distributed Consensus of Linear Multi-Agent Systems with Adaptive Dynamic Protocols *
}

\author{
Zhongkui Li, Xiangdong Liu, Wei Ren, Lihua Xie
}

September 26, 2011

\begin{abstract}
This paper considers the distributed consensus problem of multi-agent systems with general continuous-time linear dynamics. Two distributed adaptive dynamic consensus protocols are proposed, based on the relative output information of neighboring agents. One protocol assigns an adaptive coupling weight to each edge in the communication graph while the other uses an adaptive coupling weight for each node. These two adaptive protocols are designed to ensure that consensus is reached in a fully distributed fashion for any undirected connected communication graphs without using any global information. A sufficient condition for the existence of these adaptive protocols is that each agent is stabilizable and detectable. The cases with leader-follower and switching communication graphs are also studied.
\end{abstract}

Keywords: Multi-agent system, consensus, cooperative control, adaptive control, relative output.

\section{Introduction}

Consensus is an important problem in the area of cooperative control of multi-agent systems. The main idea of consensus is to develop distributed control policies that enable a group of agents to reach an agreement on certain quantities of interest. Due to its potential applications in broad areas such as spacecraft formation flying and sensor networks, the consensus problem has been extensively studied by numerous researchers from various perspectives; see [1, 2, 3, 4, 5, 6, 17] and references therein. Specifically, a general framework of the consensus problem for networks of integrators with fixed or switching topologies is proposed in [2]. The controllability of leaderfollower multi-agent systems is considered in [8] from a graph-theoretic perspective. Distributed tracking control for multi-agent consensus with an active leader is addressed in [9, 10] by using neighbor-based state estimators. Consensus of networks of double- and high-order integrators is studied in [6, 11, 12. Consensus algorithms are designed in [7, 13] for a group of agents with quantized communication links and limited data rate. In most existing studies on consensus, the agent dynamics are assumed to be first-, second-, or high-order integrators, which might be restrictive in many cases.

This paper considers the distributed consensus problem of multi-agent systems with general continuous-time linear dynamics. Previous works along this line include [14, 15, 16, 17, 18, 19,

*Zhongkui Li and Xiangdong Liu are with the School of Automation, Beijing Institute of Technology, Beijing 100081, P. R. China (e-mails: zhongkli@gmail.com,xdliu@bit.edu.cn). Wei Ren is with the Department of Electrical Engineering, University of California, Riverside, CA, 92521, USA (e-mail: ren@ee.ucr.edu). Lihua Xie is with the School of Electrical and Electronic Engineering, Nanyang Technological University, 639798, Singapore (e-mail: elhxieg@ntu.edu.sg). 
20. One common feature in [14, 15, 17, 19, 20] is that at least the smallest nonzero eigenvalue of the Laplacian matrix associated with the communication graph is required to be known for the consensus protocol design. However, the smallest nonzero eigenvalue of the Laplacian matrix is global information in the sense that each agent has to know the entire communication graph to compute it. Therefore, the consensus protocols given in [14, 15, 17, 19, 20, cannot be implemented by the agents in a fully distributed fashion, i.e., using only the local information of its own and neighbors. To overcome this limitation, an adaptive static consensus protocol is proposed in [21], which is motivated by the adaptive strategies for synchronization of complex networks in 22]. Similar adaptive schemes are presented to achieve second-order consensus with inherent nonlinear dynamics in [23]. Note that the protocols in [21, 22, 23] rely on the relative states of neighboring agents, which however might not be available in many circumstances.

In this paper, we extend [21, 23] to investigate the case where the relative outputs, rather than the relative states, of neighboring agents are accessible. Two novel distributed adaptive dynamic consensus protocols are proposed, namely, one protocol assigns an adaptive coupling weight to each edge in the communication graph while the other uses an adaptive coupling weight for each node. These two adaptive protocols are designed to ensure that consensus is reached in a fully distributed fashion for any undirected connected communication graph without using any global information. A sufficient condition for the existence of these adaptive protocols is that each agent is stabilizable and detectable. The cases with leader-follower and switching graphs are also studied. It is shown that the consensus protocol with an adaptive coupling weight for each edge is applicable to arbitrary switching connected communication graphs. It is worth mentioning that the consensus protocols in [16, 18, do not need any global information either. However, in [16] the protocol is based on the relative states of neighboring agents and the agent dynamics are restricted to be neutrally stable. In [18, the eigenvalues of the state matrix of each agent are assumed to lie in the closed left-half plane. Furthermore, the dimension of the protocol in [18] is higher than that of the consensus protocol with an adaptive coupling weight for each node in the current paper.

The rest of this paper is organized as follows. Some useful results of the graph theory are reviewed in Section 2. The consensus problems under the proposed two distributed adaptive protocols are investigated in Section 3. The consensus problem with leader-follower and switching communication graphs are studied, respectively, in Sections 4 and 5. A simulation example is presented in Section 6 to illustrate the analytical results. Section 7 concludes the paper.

\section{Notation and Graph Theory}

Let $\mathbf{R}^{n \times n}$ be the set of $n \times n$ real matrices. The superscript $T$ means the transpose for real matrices. $I_{N}$ represents the identity matrix of dimension $N$. Matrices, if not explicitly stated, are assumed to have compatible dimensions. Denote by $\mathbf{1}$ the column vector with all entries equal to one. The matrix inequality $A>B$ (respectively, $A \geq B$ ) means that $A-B$ is positive 
definite (respectively, positive semi-definite). $A \otimes B$ denotes the Kronecker product of matrices $A$ and $B \cdot \operatorname{diag}\left(A_{1}, \cdots, A_{N}\right)$ represents a block-diagonal matrix with matrices $A_{i}, i=1, \cdots, N$, on its diagonal. A matrix is Hurwitz if all of its eigenvalues have negative real parts.

A directed graph $\mathcal{G}$ is a pair $(\mathcal{V}, \mathcal{E})$, where $\mathcal{V}=\left\{v_{1}, \cdots, v_{N}\right\}$ is a nonempty finite set of nodes and $\mathcal{E} \subseteq \mathcal{V} \times \mathcal{V}$ is a set of edges, in which an edge is represented by an ordered pair of distinct nodes. For an edge $\left(v_{i}, v_{j}\right)$, node $v_{i}$ is called the parent node, node $v_{j}$ the child node, and $v_{i}$ is a neighbor of $v_{j}$. A graph with the property that $\left(v_{i}, v_{j}\right) \in \mathcal{E}$ implies $\left(v_{j}, v_{i}\right) \in \mathcal{E}$ for any $v_{i}, v_{j} \in \mathcal{V}$ is said to be undirected. A path from node $v_{i_{1}}$ to node $v_{i_{l}}$ is a sequence of ordered edges of the form $\left(v_{i_{k}}, v_{i_{k+1}}\right), k=1, \cdots, l-1$. An undirected graph is connected if there exists a path between every pair of distinct nodes, otherwise is disconnected. A directed graph contains a directed spanning tree if there exists a node called the root, which has no parent node, such that the node has directed paths to all other nodes in the graph.

The adjacency matrix $\mathcal{A}=\left[a_{i j}\right] \in \mathbf{R}^{N \times N}$ associated with the directed graph $\mathcal{G}$ is defined by $a_{i i}=0, a_{i j}=1$ if $(j, i) \in \mathcal{E}$ and $a_{i j}=0$ otherwise. The Laplacian matrix $\mathcal{L}=\left[\mathcal{L}_{i j}\right] \in \mathbf{R}^{N \times N}$ is defined as $\mathcal{L}_{i i}=\sum_{j \neq i} a_{i j}$ and $\mathcal{L}_{i j}=-a_{i j}, i \neq j$. For undirected graphs, both $\mathcal{A}$ and $\mathcal{L}$ are symmetric.

Lemma 1 [24, 2, 3].

(1) Zero is an eigenvalue of $\mathcal{L}$ with $\mathbf{1}$ as a corresponding right eigenvector, and all nonzero eigenvalues have positive real parts. Furthermore, zero is a simple eigenvalue of $\mathcal{L}$ if and only if the graph $\mathcal{G}$ has a directed spanning tree.

(2) For an undirected graph $\mathcal{G}$, the smallest nonzero eigenvalue $\lambda_{2}(\mathcal{L})$ of the Laplacian matrix $\mathcal{L}$ satisfies $\lambda_{2}(\mathcal{L})=\min _{x \neq 0,1^{T} x=0} \frac{x^{T} \mathcal{L} x}{x^{T} x}$.

\section{Consensus with Undirected Communication Graphs}

In this section, we assume that the communication graph among the agents, denoted by $\mathcal{G}$, is undirected. Consider a group of $N$ identical agents with general linear dynamics. The dynamics of the $i$-th agent are described by

$$
\begin{aligned}
& \dot{x}_{i}=A x_{i}+B u_{i}, \\
& y_{i}=C x_{i}, \quad i=1, \cdots, N,
\end{aligned}
$$

where $x_{i} \in \mathbf{R}^{n}$ is the state, $u_{i} \in \mathbf{R}^{p}$ the control input, $y_{i} \in \mathbf{R}^{q}$ the measured output, and $A, B$, $C$ are constant matrices with compatible dimensions.

In order to achieve consensus for the agents in (1), a variety of static and dynamic consensus protocols have been proposed in, e.g., [14, 15, 16, 17, 18, 19, 20]. One common feature in [14, 15, 17, 19, 20] is that at least the smallest nonzero eigenvalue $\lambda_{2}$ of the Laplacian matrix associated with $\mathcal{G}$ is required to be known for the consensus protocol design. However, $\lambda_{2}$ is global information in the sense that each agent has to know the entire graph $\mathcal{G}$ to compute it. Therefore, the consensus protocols given in [14, 15, 17, 19, 20] cannot be implemented by the agents in a fully distributed fashion, i.e., using only the local information of its own and 
neighbors. To overcome this limitation, an adaptive static consensus protocol is proposed in [21, which is motivated by the adaptive strategies for synchronization of complex networks in 222. Note that the protocols in [21, 22] are based on the relative states of neighboring agents, which however might not be available in many circumstances.

In this paper, we extend to investigate the case where each agent knows the relative outputs, rather than the relative states, of its neighbors with respect to itself. Two novel distributed adaptive dynamic consensus protocols are proposed. The first adaptive consensus protocol dynamically updates the coupling weight for each edge (i.e., the communication links between neighboring agents), which is given by

$$
\begin{aligned}
\dot{v}_{i} & =(A+B F) v_{i}+L \sum_{j=1}^{N} c_{i j} a_{i j}\left[C\left(v_{i}-v_{j}\right)-\left(y_{i}-y_{j}\right)\right], \\
\dot{c}_{i j} & =\kappa_{i j} a_{i j}\left[\begin{array}{c}
y_{i}-y_{j} \\
C\left(v_{i}-v_{j}\right)
\end{array}\right]^{T} \Gamma\left[\begin{array}{c}
y_{i}-y_{j} \\
C\left(v_{i}-v_{j}\right)
\end{array}\right], \\
u_{i} & =F v_{i}, \quad i=1, \cdots, N,
\end{aligned}
$$

where $v_{i} \in \mathbf{R}^{n}$ is the protocol state, $i=1, \cdots, N, a_{i j}$ is the $(i, j)$-th entry of the adjacency matrix $\mathcal{A}$ associated with $\mathcal{G}, c_{i j}(t)$ denotes the time-varying coupling weight for the edge $(i, j)$ with $c_{i j}(0)=c_{j i}(0), \kappa_{i j}=\kappa_{j i}$ are positive constants, and $L \in \mathbf{R}^{q \times n}, F \in \mathbf{R}^{p \times n}$, and $\Gamma \in \mathbf{R}^{2 q \times 2 q}$ are gain matrices to be determined.

The second adaptive consensus protocol assigns an adaptive coupling weight to each node (i.e., agent), described by

$$
\begin{aligned}
& \dot{\tilde{v}}_{i}=(A+B F) \tilde{v}_{i}+d_{i} L \sum_{j=1}^{N} a_{i j}\left[C\left(\tilde{v}_{i}-\tilde{v}_{j}\right)-\left(y_{i}-y_{j}\right)\right], \\
& \dot{d}_{i}=\tau_{i}\left(\sum_{j=1}^{N} a_{i j}\left[\begin{array}{c}
y_{i}-y_{j} \\
C\left(\tilde{v}_{i}-\tilde{v}_{j}\right)
\end{array}\right]^{T}\right) \Gamma\left(\sum_{j=1}^{N} a_{i j}\left[\begin{array}{c}
y_{i}-y_{j} \\
C\left(\tilde{v}_{i}-\tilde{v}_{j}\right)
\end{array}\right]\right), \\
& u_{i}=F \tilde{v}_{i}, \quad i=1, \cdots, N
\end{aligned}
$$

where $\tilde{v}_{i} \in \mathbf{R}^{n}$ is the protocol state, $i=1,2, \cdots, N, d_{i}(t)$ denotes the coupling weight for agent $i, \tau_{i}$ are positive constants, and the rest of the variables are defined as in (2).

The objective in this section is to find proper gain matrices in (2) and (3) such that the $N$ agents in (1) achieve consensus in the sense of $\lim _{t \rightarrow \infty}\left\|x_{i}(t)-x_{j}(t)\right\|=0, \forall i, j=1, \cdots, N$.

\subsection{Consensus Under Adaptive Protocol (2)}

In this section, we study the consensus problem of the agents in (1) under the adaptive protocol (2). Let $z_{i}=\left[x_{i}^{T}, v_{i}^{T}\right]^{T}, e_{i}=z_{i}-\frac{1}{N} \sum_{j=1}^{N} z_{j}, z=\left[z_{1}^{T}, \cdots, z_{N}^{T}\right]^{T}$, and $e=\left[e_{1}^{T}, \cdots, e_{N}^{T}\right]^{T}$. Then, we get $e=\left[\left(I_{N}-\frac{1}{N} \mathbf{1 1}^{T}\right) \otimes I_{2 n}\right] z$. It is easy to see that 0 is a simple eigenvalue of $I_{N}-\frac{1}{N} \mathbf{1 1}^{T}$ with 1 as a corresponding eigenvector, and 1 is the other eigenvalue with multiplicity $N-1$. Then, it follows that $e=0$ if and only if $z_{1}=\cdots=z_{N}$. Therefore, the consensus problem of 
agents (11) under the protocol (2) is solved if $e$ converges to zero. It is not difficult to obtain that $e_{i}$ and $c_{i j}$ satisfy

$$
\begin{aligned}
\dot{e}_{i} & =\mathcal{M} e_{i}+\sum_{j=1}^{N} c_{i j} a_{i j} \mathcal{H}\left(e_{i}-e_{j}\right), \\
\dot{c}_{i j} & =\kappa_{i j} a_{i j}\left(e_{i}-e_{j}\right)^{T} \mathcal{R}\left(e_{i}-e_{j}\right), \quad i=1, \cdots, N,
\end{aligned}
$$

where

$$
\begin{aligned}
\mathcal{M} & =\left[\begin{array}{cc}
A & B F \\
0 & A+B F
\end{array}\right], \quad \mathcal{H}=\left[\begin{array}{cc}
0 & 0 \\
-L C & L C
\end{array}\right], \\
\mathcal{R} & =\left(I_{2} \otimes C^{T}\right) \Gamma\left(I_{2} \otimes C\right) .
\end{aligned}
$$

The following theorem presents a sufficient condition for solving the consensus problem.

Theorem 1. Assume that the communication graph $\mathcal{G}$ is undirected and connected. Then, the $N$ agents in (11) reach consensus under the adaptive protocol (2) with $F$ satisfying that $A+B F$ is Hurwitz, $\Gamma=\left[\begin{array}{cc}I_{q} & -I_{q} \\ -I_{q} & I_{q}\end{array}\right]$, and $L=-Q^{-1} C^{T}$, where $Q>0$ is a solution to the following linear matrix inequality (LMI):

$$
A^{T} Q+Q A-2 C^{T} C<0 .
$$

Moreover, the protocol states $v_{i}, i=1, \cdots, N$, converge to zero and each coupling weight $c_{i j}$ converges to some finite steady-state value.

Proof. Consider the Lyapunov function candidate

$$
V_{1}(t)=\sum_{i=1}^{N} e_{i}^{T} \mathcal{Q} e_{i}+\sum_{i=1}^{N} \sum_{j=1, j \neq i}^{N} \frac{\left(c_{i j}-\alpha\right)^{2}}{2 \kappa_{i j}}
$$

where $\mathcal{Q}=\left[\begin{array}{cc}\varsigma P+Q & -Q \\ -Q & Q\end{array}\right], P>0$ satisfies $P(A+B F)+(A+B F)^{T} P<0$, and $\alpha$ and $\varsigma$ are positive constants to be determined later. It is easy to verify that $\mathcal{Q}>0$.

The time derivative of $V_{1}(t)$ along the trajectory of (4) can be obtained as

$$
\begin{aligned}
\dot{V}_{1}= & 2 \sum_{i=1}^{N} e_{i}^{T} \mathcal{Q} \dot{e}_{i}+\sum_{i=1}^{N} \sum_{j=1, j \neq i}^{N} \frac{c_{i j}-\alpha}{\kappa_{i j}} \dot{c}_{i j} \\
= & 2 \sum_{i=1}^{N} e_{i}^{T} \mathcal{Q}\left[\mathcal{M} e_{i}+\sum_{j=1}^{N} c_{i j} a_{i j} \mathcal{H}\left(e_{i}-e_{j}\right)\right] \\
& +\sum_{i=1}^{N} \sum_{j=1, j \neq i}^{N}\left(c_{i j}-\alpha\right) a_{i j}\left(e_{i}-e_{j}\right)^{T} \mathcal{R}\left(e_{i}-e_{j}\right) .
\end{aligned}
$$


Let $\tilde{e}_{i}=T e_{i}, i=1, \cdots, N$, with $T=\left[\begin{array}{cc}I_{q} & 0 \\ -I_{q} & I_{q}\end{array}\right]$. Then, (77) can be rewritten as

$$
\begin{aligned}
\dot{V}_{1}= & 2 \sum_{i=1}^{N} \tilde{e}_{i}^{T} \widetilde{\mathcal{Q}}\left[\widetilde{\mathcal{M}} \tilde{e}_{i}+\sum_{i=1}^{N} \sum_{j=1}^{N} c_{i j} a_{i j} \widetilde{\mathcal{H}}\left(\tilde{e}_{i}-\tilde{e}_{j}\right)\right] \\
& +\sum_{i=1}^{N} \sum_{j=1, j \neq i}^{N}\left(c_{i j}-\alpha\right) a_{i j}\left(\tilde{e}_{i}-\tilde{e}_{j}\right)^{T} \widetilde{\mathcal{R}}\left(\tilde{e}_{i}-\tilde{e}_{j}\right),
\end{aligned}
$$

where

$$
\begin{aligned}
& \widetilde{\mathcal{Q}} \triangleq T^{-T} \mathcal{Q} T^{-1}=\left[\begin{array}{cc}
\varsigma P & 0 \\
0 & Q
\end{array}\right], \quad \widetilde{\mathcal{M}} \triangleq T \mathcal{M} T^{-1}=\left[\begin{array}{cc}
A+B F & B F \\
0 & A
\end{array}\right], \\
& \widetilde{\mathcal{H}} \triangleq T \mathcal{H} T^{-1}=\left[\begin{array}{cc}
0 & 0 \\
0 & L C
\end{array}\right], \quad \widetilde{\mathcal{R}} \triangleq T^{-T} \mathcal{R} T^{-1}=\left[\begin{array}{cc}
0 & 0 \\
0 & C^{T} C
\end{array}\right] .
\end{aligned}
$$

By noting that $L=-Q^{-1} C^{T}$, we can see that

$$
\widetilde{\mathcal{Q}} \widetilde{\mathcal{H}}=\widetilde{\mathcal{R}}
$$

Because $\kappa_{i j}=\kappa_{j i}, c_{i j}(0)=c_{j i}(0)$, and $\Gamma$ is symmetric, it follows from (2) that $c_{i j}(t)=c_{j i}(t)$, $\forall t \geq 0$. Therefore, we have

$$
\begin{array}{r}
\sum_{i=1}^{N} \sum_{j=1, j \neq i}^{N}\left(c_{i j}-\alpha\right) a_{i j}\left(\tilde{e}_{i}-\tilde{e}_{j}\right)^{T} \widetilde{\mathcal{R}}\left(\tilde{e}_{i}-\tilde{e}_{j}\right) \\
=2 \sum_{i=1}^{N} \sum_{j=1}^{N}\left(c_{i j}-\alpha\right) a_{i j} \tilde{e}_{i}^{T} \widetilde{\mathcal{R}}\left(\tilde{e}_{i}-\tilde{e}_{j}\right) .
\end{array}
$$

Let $\tilde{e}=\left[\tilde{e}_{1}^{T}, \cdots, \tilde{e}_{N}^{T}\right]^{T}$. Using (10) and (9), it follows from (8) that

$$
\begin{aligned}
\dot{V}_{1} & =2 \sum_{i=1}^{N} \tilde{e}_{i}^{T} \widetilde{\mathcal{Q}} \widetilde{\mathcal{M}} \tilde{e}_{i}-2 \sum_{i=1}^{N} \sum_{j=1}^{N} \alpha a_{i j} \tilde{e}_{i}^{T} \widetilde{\mathcal{R}}\left(\tilde{e}_{i}-\tilde{e}_{j}\right) \\
& =\tilde{e}^{T}\left[I_{N} \otimes\left(\widetilde{\mathcal{Q}} \widetilde{\mathcal{M}}+\widetilde{\mathcal{M}}^{T} \widetilde{\mathcal{Q}}\right)-2 \alpha \mathcal{L} \otimes \widetilde{\mathcal{R}}\right] \tilde{e}
\end{aligned}
$$

where $\mathcal{L}$ is the Laplacian matrix associated with $\mathcal{G}$.

By the definitions of $e$ and $\tilde{e}$, it is easy to see that $\left(\mathbf{1}^{T} \otimes I\right) \tilde{e}=\left(\mathbf{1}^{T} \otimes T\right) e=0$. Because $\mathcal{G}$ is connected, it then follows from Lemma 1 that

$$
\tilde{e}^{T}(\mathcal{L} \otimes I) \tilde{e} \geq \lambda_{2}(\mathcal{L}) \tilde{e}^{T} \tilde{e}
$$

where $\lambda_{2}(\mathcal{L})$ is the smallest nonzero eigenvalue of $\mathcal{L}$. Therefore, we can get from (11) that

$$
\dot{V}_{1} \leq \tilde{e}^{T}\left[I_{N} \otimes\left(\widetilde{\mathcal{Q}} \widetilde{\mathcal{M}}+\widetilde{\mathcal{M}}^{T} \widetilde{\mathcal{Q}}-2 \alpha \lambda_{2}(\mathcal{L}) \widetilde{\mathcal{R}}\right)\right] \tilde{e}
$$

Note that

$$
\begin{aligned}
& \widetilde{\mathcal{Q}} \widetilde{\mathcal{M}}+\widetilde{\mathcal{M}}^{T} \widetilde{\mathcal{Q}}-2 \alpha \lambda_{2}(\mathcal{L}) \widetilde{\mathcal{R}} \\
& =\left[\begin{array}{cc}
\varsigma\left(P(A+B F)+(A+B F)^{T} P\right) & \varsigma P B F \\
\varsigma F^{T} B^{T} P & Q A+A^{T} Q-2 \alpha \lambda_{2}(\mathcal{L}) C^{T} C
\end{array}\right] .
\end{aligned}
$$


By choosing $\alpha$ sufficiently large such that $\alpha \lambda_{2}(\mathcal{L}) \geq 1$, it follows from (5) that $Q A+A^{T} Q-$ $2 \alpha \lambda_{2}(\mathcal{L}) C^{T} C<0$. Then, choosing $\varsigma>0$ sufficiently small, and in virtue of Schur Complement Lemma [25], we can obtain from (14) that

$$
\widetilde{\mathcal{Q}} \widetilde{\mathcal{M}}+\widetilde{\mathcal{M}}^{T} \widetilde{\mathcal{Q}}-2 \alpha \lambda_{2}(\mathcal{L}) \widetilde{\mathcal{R}}<0 .
$$

Therefore, $\dot{V}_{1} \leq 0$.

Since $\dot{V}_{1} \leq 0, V_{1}(t)$ is bounded, implying that each $c_{i j}$ is also bounded. By noting that $\mathcal{R}$ is positive semi-definite, we can see from (44) that $c_{i j}$ is monotonically increasing. Then, it follows that each coupling weight $c_{i j}$ converges to some finite value. Note that $\dot{V}_{1} \equiv 0$ implies that $\tilde{e}=0$ and $e=0$. Hence, by LaSalle's Invariance principle [26], it follows that $e(t) \rightarrow 0$, as $t \rightarrow \infty$. That is, the consensus problem is solved. By (2) and noting the fact that $A+B F$ is Hurwitz, it is easy to see that the protocol states $v_{i}, i=1, \cdots, N$, converge to zero.

Remark 1. As shown in [14], a necessary and sufficient condition for the existence of a $Q>0$ to the LMI (5) is that $(A, C)$ is detectable. Therefore, a sufficient condition for the existence of a protocol (2) satisfying Theorem 1 is that $(A, B, C)$ is stabilizable and detectable. It is worth noting that a favorable feature of (2) is that its gain matrices $F, L$ and $\Gamma$ can be independently designed.

\subsection{Consensus Under Adaptive Protocol (3)}

This section considers the consensus problem of the agents in (11) under the adaptive protocol (3). Let $\tilde{z}_{i}=\left[x_{i}^{T}, \tilde{v}_{i}^{T}\right]^{T}, \zeta_{i}=\tilde{z}_{i}-\frac{1}{N} \sum_{j=1}^{N} \tilde{z}_{j}$, and $\zeta=\left[\zeta_{1}^{T}, \cdots, \zeta_{N}^{T}\right]^{T}$. As shown in the last subsection, the consensus problem of agents (11) under the protocol (3) is solved if $\zeta$ converges to zero. We can obtain that $\zeta_{i}$ and $d_{i}$ satisfy the following dynamics:

$$
\begin{aligned}
& \dot{\zeta}_{i}=\mathcal{M} \zeta_{i}+d_{i} \sum_{j=1}^{N} a_{i j} \mathcal{H}\left(\zeta_{i}-\zeta_{j}\right)-\frac{1}{N} \sum_{k=1}^{N} d_{k} \sum_{j=1}^{N} a_{k j} \mathcal{H}\left(\zeta_{k}-\zeta_{j}\right), \\
& \dot{d}_{i}=\tau_{i}\left[\sum_{j=1}^{N} a_{i j}\left(\zeta_{i}-\zeta_{j}\right)^{T}\right] \mathcal{R}\left[\sum_{j=1}^{N} a_{i j}\left(\zeta_{i}-\zeta_{j}\right)\right], \quad i=1, \cdots, N,
\end{aligned}
$$

where $\mathcal{M}, \mathcal{H}, \mathcal{R}$ are defined in (4). Let $D(t)=\operatorname{diag}\left(d_{1}(t), \cdots, d_{N}(t)\right)$. Then, the first equation in (15) can be rewritten into a compact form as

$$
\dot{\zeta}=\left[I_{N} \otimes \mathcal{M}+\left(\left(I_{N}-\frac{1}{N} \mathbf{1 1}^{T}\right) D \mathcal{L}\right) \otimes \mathcal{H}\right] \zeta,
$$

where $\mathcal{L}$ is the Laplacian matrix associated with $\mathcal{G}$.

Theorem 2. Assume that the communication graph $\mathcal{G}$ is undirected and connected. Then, the $N$ agents in (11) reach consensus under the protocol (3) with $F, L$, and $\Gamma$ given as in Theorem 1. Moreover, the protocol states $\tilde{v}_{i}, i=1, \cdots, N$, converge to zero and each coupling weight $\tilde{d}_{i}$ converges to some finite steady-state value.

Proof. Consider the Lyapunov function candidate

$$
V_{2}=\zeta^{T}(\mathcal{L} \otimes \mathcal{Q}) \zeta+\sum_{i=1}^{N} \frac{\left(d_{i}-\beta\right)^{2}}{2 \tau_{i}},
$$


where $\mathcal{Q}$ is defined in (6), and $\beta$ is a positive constant to be determined later. For a connected graph $\mathcal{G}$, it follows from Lemma 1 and the definition of $\zeta$ that $\zeta^{T}(\mathcal{L} \otimes \mathcal{Q}) \zeta \geq \lambda_{2}(\mathcal{L}) \zeta^{T}\left(I_{N} \otimes \mathcal{Q}\right) \zeta$. Therefore, it is easy to see that $\Omega_{c}=\left\{\zeta, d_{i} \mid V_{2} \leq c\right\}$ is compact for any positive $c$.

Following similar steps to those in the proof of Theorem 1, we can obtain the time derivative of $V_{2}$ along the trajectory of (16) as

$$
\begin{aligned}
\dot{V}_{2} & =2 \zeta^{T}(\mathcal{L} \otimes \mathcal{Q}) \zeta+\sum_{i=1}^{N} \frac{d_{i}-\beta}{\tau_{i}} \dot{d}_{i} \\
& =2 \zeta^{T}\left[\mathcal{L} \otimes \mathcal{Q} \mathcal{M}+\left(\mathcal{L} D \mathcal{L}-\mathcal{L} 11^{T} D \mathcal{L}\right) \otimes \mathcal{Q H}\right] \zeta+\sum_{i=1}^{N} \frac{d_{i}-\beta}{\tau_{i}} \dot{d}_{i} \\
& =2 \tilde{\zeta}^{T}[\mathcal{L} \otimes \widetilde{\mathcal{Q}} \widetilde{\mathcal{M}}+(\mathcal{L} D \mathcal{L}) \otimes \widetilde{\mathcal{R}}] \tilde{\zeta}+\sum_{i=1}^{N} \frac{d_{i}-\beta}{\tau_{i}} \dot{d}_{i} .
\end{aligned}
$$

where $\tilde{\zeta} \triangleq\left[\tilde{\zeta}_{1}^{T}, \cdots, \tilde{\zeta}_{N}^{T}\right]^{T}=\left(I_{N} \otimes T\right) \zeta$ with $T=\left[\begin{array}{cc}I_{q} & 0 \\ -I_{q} & I_{q}\end{array}\right]$, and $\widetilde{\mathcal{Q}}, \widetilde{\mathcal{M}}, \widetilde{\mathcal{R}}$ are the same as in (8). Observe that

$$
\tilde{\zeta}^{T}[(\mathcal{L} D \mathcal{L}) \otimes \widetilde{\mathcal{R}}] \tilde{\zeta}=\sum_{i=1}^{N} d_{i}\left[\sum_{j=1}^{N} a_{i j}\left(\tilde{\zeta}_{i}-\tilde{\zeta}_{j}\right)^{T}\right] \widetilde{\mathcal{R}}\left[\sum_{j=1}^{N} a_{i j}\left(\tilde{\zeta}_{i}-\tilde{\zeta}_{j}\right)\right]
$$

Moreover, the second equation in (15) can be rewritten as

$$
\dot{d}_{i}=\tau_{i}\left[\sum_{j=1}^{N} a_{i j}\left(\tilde{\zeta}_{i}-\tilde{\zeta}_{j}\right)^{T}\right] \widetilde{\mathcal{R}}\left[\sum_{j=1}^{N} a_{i j}\left(\tilde{\zeta}_{i}-\tilde{\zeta}_{j}\right)\right]
$$

Substituting (19) and (201) into (18) yields

$$
\begin{aligned}
\dot{V}_{2} & =2 \tilde{\zeta}^{T}(\mathcal{L} \otimes \widetilde{\mathcal{Q}} \widetilde{\mathcal{M}}) \tilde{\zeta}-2 \beta \sum_{i=1}^{N}\left[\sum_{j=1}^{N} a_{i j}\left(\tilde{\zeta}_{i}-\tilde{\zeta}_{j}\right)^{T}\right] \widetilde{\mathcal{R}}\left[\sum_{j=1}^{N} a_{i j}\left(\tilde{\zeta}_{i}-\tilde{\zeta}_{j}\right)\right] \\
& =\tilde{\zeta}^{T}\left[\mathcal{L} \otimes\left(\widetilde{\mathcal{Q}} \widetilde{\mathcal{M}}+\widetilde{\mathcal{M}}^{T} \widetilde{\mathcal{Q}}^{T}\right)-2 \beta \mathcal{L}^{2} \otimes \widetilde{\mathcal{R}}\right] \tilde{\zeta}
\end{aligned}
$$

Because $\mathcal{G}$ is connected, it follows from Lemma 1 that zero is a simple eigenvalue of $\mathcal{L}$ and all the other eigenvalues are positive. Let $U=\left[\begin{array}{ll}\mathbf{1} & Y_{1}\end{array}\right]$ and $U^{T}=\left[\begin{array}{c}\frac{1^{T}}{N} \\ Y_{2}\end{array}\right]$, with $Y_{1} \in \mathbf{R}^{N \times(N-1)}$, $Y_{2} \in \mathbf{R}^{(N-1) \times N}$, be such unitary matrices that $U^{T} \mathcal{L} U=\Lambda \triangleq \operatorname{diag}\left(0, \lambda_{2}, \cdots, \lambda_{N}\right)$, where $\lambda_{2} \leq$ $\cdots \leq \lambda_{N}$ are the nonzero eigenvalues of $\mathcal{L}$. Let $\bar{\zeta} \triangleq\left[\bar{\zeta}_{1}^{T}, \cdots, \bar{\zeta}_{N}^{T}\right]^{T}=\left(U^{T} \otimes I\right) \tilde{\zeta}$. By the definitions of $\zeta$ and $\tilde{\zeta}$, it is easy to see that $\bar{\zeta}_{1}=\left(\mathbf{1}^{T} \otimes T\right) \zeta=0$. Then, it follows from (21) that

$$
\begin{aligned}
\dot{V}_{2} & =\bar{\zeta}^{T}\left[\Lambda \otimes\left(\widetilde{\mathcal{Q}} \widetilde{\mathcal{M}}+\widetilde{\mathcal{M}}^{T} \widetilde{\mathcal{Q}}^{T}\right)-2 \beta \Lambda^{2} \otimes \widetilde{\mathcal{R}}\right] \bar{\zeta} \\
& =\sum_{i=2}^{N} \lambda_{i} \bar{\zeta}_{i}^{T}\left(\widetilde{\mathcal{Q}} \widetilde{\mathcal{M}}+\widetilde{\mathcal{M}}^{T} \widetilde{\mathcal{Q}}^{T}-2 \beta \lambda_{i} \widetilde{\mathcal{R}}\right) \bar{\zeta}_{i}
\end{aligned}
$$

As shown in the proof of Theorem 1 , by choosing $\beta$ sufficiently large such that $\beta \lambda_{2}(\mathcal{L}) \geq 1$ and $\varsigma>0$ sufficiently small, we can obtain from (22) that $\dot{V}_{2} \leq 0$. Note that $\dot{V}_{2} \equiv 0$ that $\bar{\zeta}_{i}=0$, $i=2, \cdots, N$, which, together with $\bar{\zeta}_{1}=0$, further implies that $\zeta=0$. Therefore, it follows from 
Lasalle's Invariance principle that $\zeta \rightarrow 0$, as $t \rightarrow \infty$. The convergence of $d_{i}$ and $\tilde{v}_{i}, i=1, \cdots, N$, can be shown by following similar steps in the proof of Theorem 1, which is omitted here for brevity.

Remark 2. Different from the previous adaptive schemes in [21, 22, 23], which are based on the relative state information, the proposed adaptive protocols (2) and (3) rely on the relative outputs of neighboring agents. Contrary to the protocols in [14, 15, 17, 19, 20], the adaptive protocols (2) and (3) can be computed and implemented by each agent in a fully distributed fashion without using any global information. It is worth mentioning that the consensus protocols in [16, 18] do not need any global information either. However, in [16] the protocol is based on the relative states of neighboring agents and the agent dynamics are restricted to be neutrally stable. In [18], the eigenvalues of the state matrix of each agent are assumed to lie in the closed left-half plane. Furthermore, the dimension of the protocol in [18] is higher than that of the adaptive protocol (3) .

Remark 3. Some comparisons between the adaptive consensus protocols (21) and (3) are now briefly discussed. The dimension of the adaptive protocol (2) is proportional to the number of edges in the communication graph. Since the number of edges is usually larger than the number of nodes in a connected graph, the dimension of the protocol (2) are generally higher than that of (3). On the other hand, the adaptive protocol (2) are applicable to the case with switching communication graphs, which will be shown in Section 5 .

\section{Consensus with Leader-Follower Communication Graphs}

The section extends to consider the case where the $N$ agents in (11) maintain a leader-follower communication graph $\mathcal{G}$. Without loss of generality, assume that the agent indexed by 1 is the leader whose control input $u_{1}=0$ and the agents indexed by $2, \cdots, N$, are followers. The leader does not receive any information from the followers, i.e., it has no neighbor, while each follower can obtain the relative outputs with respect to its neighbors.

In the sequel, the following assumption is needed.

Assumption 1. The subgraph associated with the followers is undirected and the graph $\mathcal{G}$ contains a directed spanning tree with the leader as the root.

Denote by $\mathcal{L}$ the Laplacian matrix associated with $\mathcal{G}$. Because the leader has no neighbors, $\mathcal{L}$ can be partitioned as

$$
\mathcal{L}=\left[\begin{array}{cc}
0 & 0_{1 \times(N-1)} \\
\mathcal{L}_{2} & \mathcal{L}_{1}
\end{array}\right],
$$

where $\mathcal{L}_{2} \in \mathbf{R}^{(N-1) \times 1}$ and $\mathcal{L}_{1} \in \mathbf{R}^{(N-1) \times(N-1)}$ is symmetric.

It is said that the leader-follower consensus problem is solved if the states of the followers converge to the state of the leader, i.e., $\lim _{t \rightarrow \infty}\left\|x_{i}(t)-x_{1}(t)\right\|=0, \forall i=2, \cdots, N$.

Based on the relative output information of neighboring agents, the first adaptive consensus 
protocol with a dynamic coupling weight for each edge is proposed for the followers as follows:

$$
\begin{aligned}
\dot{\bar{v}}_{i} & =(A+B F) \bar{v}_{i}+L \sum_{j=1}^{N} d_{i j} a_{i j}\left[C\left(\bar{v}_{i}-\bar{v}_{j}\right)-\left(y_{i}-y_{j}\right)\right], \\
\dot{d}_{i j} & =\epsilon_{i j} a_{i j}\left[\begin{array}{c}
y_{i}-y_{j} \\
C\left(\bar{v}_{i}-\bar{v}_{j}\right)
\end{array}\right]^{T} \Gamma\left[\begin{array}{c}
y_{i}-y_{j} \\
C\left(\bar{v}_{i}-\bar{v}_{j}\right)
\end{array}\right], \\
u_{i} & =F \bar{v}_{i}, \quad i=2, \cdots, N,
\end{aligned}
$$

where $\bar{v}_{i} \in \mathbf{R}^{n}$ is the protocol state, $i=2, \cdots, N, \bar{v}_{1} \in \mathbf{R}^{n}$ is generated by $\dot{\bar{v}}_{1}=(A+B F) \bar{v}_{1}, a_{i j}$ is the $(i, j)$-th entry of the adjacency matrix $\mathcal{A}$ of $\mathcal{G}, d_{i j}$ is the coupling weight associated with the edge $(j, i)$ with $d_{i j}(0)=d_{j i}(0)$ for $i, j=2, \cdots, N, \epsilon_{i j}=\epsilon_{j i}$ are positive constants, $L \in \mathbf{R}^{q \times n}$, $F \in \mathbf{R}^{p \times n}$, and $\Gamma \in \mathbf{R}^{2 q \times 2 q}$.

Theorem 3. For any graph $\mathcal{G}$ satisfying Assumption 1, the $N$ agents in (1) reach leaderfollower consensus under the protocol (24) with $F, L$, and $\Gamma$ given as in Theorem 1. Meanwhile, the protocol states $\bar{v}_{i}, i=2, \cdots, N$, converge to zero and the coupling weights $d_{i j}$ converge to finite steady-state values.

Proof. Let $\xi_{i}=\left[\begin{array}{c}x_{i}-x_{1} \\ \bar{v}_{i}-\bar{v}_{1}\end{array}\right], i=2, \cdots, N$. Then, we can get from (11) and (24) that

$$
\begin{aligned}
\dot{\xi}_{i} & =\mathcal{M} \xi_{i}+\sum_{j=2}^{N} d_{i j} a_{i j} \mathcal{H}\left(\xi_{i}-\xi_{j}\right)+d_{i 1} a_{i 1} \mathcal{H} \xi_{i}, \\
\dot{d}_{i 1} & =\epsilon_{i 1} a_{i 1} \xi_{i}^{T} \mathcal{R} \xi_{i}, \\
\dot{d}_{i j} & =\epsilon_{i j} a_{i j}\left(\xi_{i}-\xi_{j}\right)^{T} \mathcal{R}\left(\xi_{i}-\xi_{j}\right), i=2, \cdots, N,
\end{aligned}
$$

where $\mathcal{M}, \mathcal{H}$ and $\mathcal{R}$ are the same as in (4). Clearly, the leader-follower consensus problem of (11) is solved by (24) if the states $\xi_{i}$ of (25) converge to zero.

Consider the Lyapunov function candidate

$$
V_{3}=\sum_{i=2}^{N} \xi_{i}^{T} \mathcal{Q} \xi_{i}+\sum_{i=2}^{N} \sum_{j=2, j \neq i}^{N} \frac{\left(d_{i j}-\gamma\right)^{2}}{2 \epsilon_{i j}}+\sum_{i=2}^{N} \frac{\left(d_{i 1}-\gamma\right)^{2}}{\epsilon_{i 1}},
$$

where $\gamma$ is a positive constant and $\mathcal{Q}$ is defined in ([6). Following similar steps to those in the proof of Theorem 1, the time derivative of $V_{2}$ along the trajectory of (25) can be obtained as

$$
\begin{aligned}
\dot{V}_{3}= & 2 \sum_{i=2}^{N} \xi_{i}^{T} \mathcal{Q} \dot{\xi}_{i}+\sum_{i=2}^{N} \sum_{j=2, j \neq i}^{N} \frac{d_{i j}-\beta}{\epsilon_{i j}} \dot{d}_{i j}+\sum_{i=2}^{N} \frac{2\left(d_{i 1}-\gamma\right)}{\epsilon_{i 1}} \dot{d}_{i 1} \\
= & 2 \sum_{i=2}^{N} \tilde{\xi}_{i}^{T} \widetilde{\mathcal{Q}}\left[\widetilde{\mathcal{M}} \tilde{\xi}_{i}+\sum_{j=2}^{N} d_{i j} a_{i j} \widetilde{\mathcal{H}}\left(\tilde{\xi}_{i}-\tilde{\xi}_{j}\right)+d_{i 1} a_{i 1} \widetilde{\mathcal{H}}_{i}\right] \\
& +\sum_{i=2}^{N} \sum_{j=2, j \neq i}^{N}\left(d_{i j}-\gamma\right) a_{i j}\left(\tilde{\xi}_{i}-\tilde{\xi}_{j}\right)^{T} \widetilde{\mathcal{R}}\left(\tilde{\xi}_{i}-\tilde{\xi}_{j}\right)+2 \sum_{i=2}^{N}\left(d_{i 1}-\gamma\right) a_{i 1} \tilde{\xi}_{i}^{T} \widetilde{\mathcal{R}}_{i},
\end{aligned}
$$

where $\tilde{\xi}_{i}=T \xi_{i}$ with $T=\left[\begin{array}{cc}I_{q} & 0 \\ -I_{q} & I_{q}\end{array}\right]$, and $\widetilde{\mathcal{Q}}, \widetilde{\mathcal{M}}, \widetilde{\mathcal{H}}, \widetilde{\mathcal{R}}$ are defined in (8). 
Since the subgraph associated with the $N-1$ followers is undirected, we have

$$
\begin{array}{r}
\sum_{i=2}^{N} \sum_{j=2, j \neq i}^{N}\left(d_{i j}-\gamma\right) a_{i j}\left(\tilde{\xi}_{i}-\tilde{\xi}_{j}\right)^{T} \widetilde{\mathcal{R}}\left(\tilde{\xi}_{i}-\tilde{\xi}_{j}\right) \\
=2 \sum_{i=2}^{N} \sum_{j=2}^{N}\left(d_{i j}-\gamma\right) a_{i j} \tilde{\xi}_{i}^{T} \widetilde{\mathcal{R}}\left(\tilde{\xi}_{i}-\tilde{\xi}_{j}\right)
\end{array}
$$

Letting $\tilde{\xi}=\left[\tilde{\xi}_{2}^{T}, \cdots, \tilde{\xi}_{N}^{T}\right]^{T}$, it then follows from (27) that

$$
\begin{aligned}
\dot{V}_{3} & =2 \sum_{i=2}^{N} \tilde{\xi}_{i}^{T} \widetilde{\mathcal{Q}} \widetilde{\mathcal{M}} \tilde{\xi}_{i}-2 \gamma \sum_{i=2}^{N} \sum_{j=2}^{N} a_{i j} \tilde{\xi}_{i}^{T} \widetilde{\mathcal{R}}\left(\tilde{\xi}_{i}-\tilde{\xi}_{j}\right)-2 \gamma \sum_{i=2}^{N} a_{i 1} \tilde{\xi}_{i}^{T} \widetilde{\mathcal{R}} \tilde{\xi}_{i} \\
& =\tilde{\xi}^{T}\left[I_{N-1} \otimes\left(\widetilde{\mathcal{Q}} \widetilde{\mathcal{M}}+\widetilde{\mathcal{M}}^{T} \widetilde{\mathcal{Q}}\right)-2 \gamma \mathcal{L}_{1} \otimes \widetilde{\mathcal{R}}\right] \tilde{\xi},
\end{aligned}
$$

where $\mathcal{L}_{1}$ is defined in (23).

For any graph $\mathcal{G}$ satisfying Assumption 1, it follows from Lemma 1 and (23) that $\mathcal{L}_{1}$ is positive definite. Thus,

$$
\tilde{\xi}^{T}\left(\mathcal{L}_{1} \otimes \widetilde{\mathcal{R}}\right) \tilde{\xi} \geq \lambda_{2}(\mathcal{L}) \tilde{\xi}^{T}\left(I_{N-1} \otimes \widetilde{\mathcal{R}}\right) \tilde{\xi}
$$

where $\lambda_{2}(\mathcal{L})$ is the smallest eigenvalue of $\mathcal{L}_{1}$. Then, we have

$$
\dot{V}_{3} \leq \tilde{\xi}^{T}\left[I_{N-1} \otimes\left(\widetilde{\mathcal{Q}} \widetilde{\mathcal{M}}+\widetilde{\mathcal{M}}^{T} \widetilde{\mathcal{Q}}-2 \gamma \lambda_{2}(\mathcal{L}) \widetilde{\mathcal{R}}\right)\right] \tilde{\xi}
$$

The rest of the proof is similar to that of Theorem 1, which is omitted for brevity.

Corresponding to (3), the second adaptive consensus protocol with a time-varying coupling weight for each follower is proposed as

$$
\begin{aligned}
& \dot{\hat{v}}_{i}=(A+B F) \hat{v}_{i}+\hat{d}_{i} L \sum_{j=1}^{N} a_{i j}\left[C\left(\hat{v}_{i}-\hat{v}_{j}\right)-\left(y_{i}-y_{j}\right)\right], \\
& \dot{\hat{d}}_{i}=\epsilon_{i}\left(\sum_{j=1}^{N} a_{i j}\left[\begin{array}{c}
y_{i}-y_{j} \\
C\left(\hat{v}_{i}-\hat{v}_{j}\right)
\end{array}\right]^{T}\right) \Gamma\left(\sum_{j=1}^{N} a_{i j}\left[\begin{array}{c}
y_{i}-y_{j} \\
C\left(\hat{v}_{i}-\hat{v}_{j}\right)
\end{array}\right]\right), \\
& u_{i}=F \hat{v}_{i}, \quad i=2, \cdots, N
\end{aligned}
$$

where $\hat{v}_{i} \in \mathbf{R}^{n}$ is the protocol state, $i=2, \cdots, N, \hat{v}_{1} \in \mathbf{R}^{n}$ is generated by $\dot{\hat{v}}_{1}=(A+B F) \hat{v}_{1}, \hat{d}_{i}$ denotes the coupling weight associated with follower $i$, and $\epsilon_{i}$ are positive constants.

Theorem 4. For any graph $\mathcal{G}$ satisfying Assumption 1 , the $N$ agents in (11) reach leaderfollower consensus under the protocol (30) with with $F, L$, and $\Gamma$ given as in Theorem 1. Meanwhile, the protocol states $\hat{v}_{i}, i=2, \cdots, N$, converge to zero and each coupling weight $\hat{d}_{i}$ converges to some finite steady-state value.

Proof. Let $\varrho_{i}=\left[\begin{array}{c}x_{i}-x_{1} \\ \hat{v}_{i}-\hat{v}_{1}\end{array}\right], i=2, \cdots, N$. Clearly, the leader-follower consensus problem of (11) under (30) is solved if $\varrho_{i}, i=2, \cdots, N$, converge to zero. It is easy to see that $\varrho_{i}$ and $\hat{d}_{i}$ 
satisfy

$$
\begin{aligned}
& \dot{\varrho}_{i}=\mathcal{M} \varrho_{i}+\hat{d}_{i} \mathcal{H}\left[\sum_{j=2}^{N} a_{i j}\left(\varrho_{i}-\varrho_{j}\right)+a_{i 1} \varrho_{i}\right], \\
& \dot{\hat{d}}_{i}=\epsilon_{i}\left[\sum_{j=2}^{N} a_{i j}\left(\varrho_{i}-\varrho_{j}\right)+a_{i 1} \varrho_{i}\right]^{T} \mathcal{R}\left[\sum_{j=2}^{N} a_{i j}\left(\varrho_{i}-\varrho_{j}\right)+a_{i 1} \varrho_{i}\right], i=2, \cdots, N .
\end{aligned}
$$

Consider the Lyapunov function candidate

$$
V_{4}=\sum_{i=2}^{N} \varrho_{i}^{T} \mathcal{Q} \varrho_{i}+\sum_{i=2}^{N} \frac{\left(\hat{d}_{i}-\sigma\right)^{2}}{2 \epsilon_{i}},
$$

where $\sigma$ is a positive constant and $\mathcal{Q}$ is defined in (6). The rest of the proof can be completed by following similar steps in proving Theorems 2 and 3.

\section{Extensions to Switching Communication Graphs}

In the last sections, the communication graph is assumed to be fixed throughout the whole process. However, the communication graph may change with time in many practical situations due to various reasons, such as communication constraints, link variations, etc. In this section, the consensus problem under the adaptive protocol (2) with switching communication graphs will be considered.

Denote by $\mathscr{G}_{N}$ the set of all possible undirected connected graphs with $N$ nodes. Let $\sigma(t)$ : $[0, \infty) \rightarrow \mathscr{P}$ be a piecewise constant switching signal with switching times $t_{0}, t_{1}, \cdots$, and $\mathscr{P}$ be the index set associated with the elements of $\mathscr{G}_{N}$, which is clearly finite. The communication graph at time $t$ is denoted by $\mathcal{G}_{\sigma(t)}$. Accordingly, (2) becomes

$$
\begin{aligned}
\dot{v}_{i} & =(A+B F) v_{i}+L \sum_{j=1}^{N} c_{i j} a_{i j}(t)\left[C\left(v_{i}-v_{j}\right)-\left(y_{i}-y_{j}\right)\right], \\
\dot{c}_{i j} & =\kappa_{i j} a_{i j}(t)\left[\begin{array}{c}
y_{i}-y_{j} \\
C\left(v_{i}-v_{j}\right)
\end{array}\right]^{T} \Gamma\left[\begin{array}{c}
y_{i}-y_{j} \\
C\left(v_{i}-v_{j}\right)
\end{array}\right], \\
u_{i} & =F v_{i}, \quad i=1, \cdots, N,
\end{aligned}
$$

where $a_{i j}(t)$ is the $(i, j)$-th entry of the adjacency matrix associated with $\mathcal{G}_{\sigma(t)}$ and the rest of the variables are the same as in (2).

Theorem 5. For arbitrary switching communication graphs $\mathcal{G}_{\sigma(t)}$ belonging to $\mathscr{G}_{N}$, the $N$ agents in (11) reach consensus under the protocol (33) with $F, L$ and $\Gamma$ given as in Theorem 1. Besides, the protocol states $v_{i}, i=1, \cdots, N$, converge to zero and the coupling weights $c_{i j}$ converge to some finite values.

Proof. Let $e_{i}=x_{i}-\frac{1}{N} \sum_{j=1}^{N} x_{j}, i=1, \cdots, N$, and $e=\left[e_{1}^{T}, \cdots, e_{N}^{T}\right]^{T}$. By following similar steps to those in the proof of Theorem 1, the consensus problem of the agents (1) under the 
protocol (33) is solved if $e$ converges to zero. Clearly $e_{i}$ and $\tilde{c}_{i j}$ satisfy

$$
\begin{aligned}
\dot{e}_{i} & =\mathcal{M} e_{i}+\sum_{j=1}^{N}\left(\tilde{c}_{i j}+\delta\right) a_{i j}(t) \mathcal{H}\left(e_{i}-e_{j}\right), \\
\dot{\tilde{c}}_{i j} & =\kappa_{i j} a_{i j}(t)\left(e_{i}-e_{j}\right)^{T} \mathcal{R}\left(e_{i}-e_{j}\right), \quad i=1, \cdots, N,
\end{aligned}
$$

where $c_{i j}=\tilde{c}_{i j}+\delta, \delta$ is a positive scalar, and $\mathcal{M}, \mathcal{H}$ and $\mathcal{R}$ are defined as in (4).

Take a common Lyapunov function candidate as

$$
V_{5}(t)=\sum_{i=1}^{N} e_{i}^{T} \mathcal{Q} e_{i}+\sum_{i=1}^{N} \sum_{j=1, j \neq i}^{N} \frac{\tilde{c}_{i j}^{2}}{2 \kappa_{i j}},
$$

where $\mathcal{Q}$ is defined in (6). The time derivative of $V_{5}$ along the trajectory of (34) can be obtained as

$$
\begin{aligned}
\dot{V}_{5}= & 2 \sum_{i=1}^{N} e_{i}^{T} \mathcal{Q} \dot{e}_{i}+\sum_{i=1}^{N} \sum_{j=1, j \neq i}^{N} \frac{\tilde{c}_{i j}}{\kappa_{i j}} \dot{\tilde{c}}_{i j} \\
= & 2 \sum_{i=1}^{N} e_{i}^{T} \widetilde{\mathcal{Q}}\left[\widetilde{\mathcal{M}} e_{i}+\sum_{j=1}^{N}\left(\tilde{c}_{i j}+\delta\right) a_{i j}(t) \widetilde{\mathcal{H}}\left(e_{i}-e_{j}\right)\right] \\
& +\sum_{i=1}^{N} \sum_{j=1, j \neq i}^{N} c_{i j} a_{i j}(t)\left(e_{i}-e_{j}\right)^{T} \widetilde{\mathcal{R}}\left(e_{i}-e_{j}\right) \\
= & \tilde{e}^{T}\left[I_{N} \otimes(\widetilde{\mathcal{Q}} \widetilde{\mathcal{M}}+\widetilde{\mathcal{M}} T \widetilde{\mathcal{Q}})-2 \delta \mathcal{L}_{\sigma(t)} \otimes \widetilde{\mathcal{R}}\right] \tilde{e},
\end{aligned}
$$

where (10) has been used to obtain the last equality, $\mathcal{L}_{\sigma(t)}$ is the Laplacian matrix associated with $\mathcal{G}_{\sigma(t)}$, and the rest of the variables are the same as in (8).

Since $\mathcal{G}_{\sigma(t)}$ is connected and $\left(\mathbf{1}^{T} \otimes I\right) \tilde{e}=0$, it is easy to see that

$$
\tilde{e}^{T}\left(\mathcal{L}_{\sigma(t)} \otimes I\right) \tilde{e} \geq \lambda_{2}^{\min } \tilde{e}^{T} \tilde{e}
$$

where $\lambda_{2}^{\min } \triangleq \min _{\mathcal{G}_{\sigma(t)} \in \mathscr{G}_{N}}\left\{\lambda_{2}\left(\mathcal{L}_{\sigma(t)}\right)\right\}$ denotes the minimum of the smallest nonzero eigenvalues of $\mathcal{L}_{\sigma(t)}$ for all $\mathcal{G}_{\sigma(t)} \in \mathscr{G}_{N}$. Therefore, we can get from (36) that

$$
\begin{aligned}
\dot{V}_{5} & \leq \tilde{e}^{T}\left[I_{N} \otimes\left(\widetilde{\mathcal{Q}} \widetilde{\mathcal{M}}+\widetilde{\mathcal{M}}^{T} \widetilde{\mathcal{Q}}-2 \delta \lambda_{2}^{\min } \widetilde{\mathcal{R}}\right)\right] \tilde{e} \\
& \triangleq W(\tilde{e}) .
\end{aligned}
$$

As shown in the proof of Theorem 1 , by choosing $\delta$ sufficiently large such that $\delta \lambda_{2}^{\min } \geq 1$ and $\gamma>0$ sufficiently small, we have $\widetilde{\mathcal{Q}} \widetilde{\mathcal{M}}+\widetilde{\mathcal{M}}^{T} \widetilde{\mathcal{Q}}-2 \delta \lambda_{2}^{\min } \widetilde{\mathcal{R}}<0$. Therefore, $\dot{V}_{5} \leq 0$, implying that $V_{5}$ is bounded. Consequently, $c_{i j}, i, j=1, \cdots, N$, are bounded. By noting (34), each $\tilde{c}_{i j}$ is monotonically increasing. It then follows that each $\tilde{c}_{i j}$ converges to some finite value. Thus, the coupling weights $c_{i j}$ converge to finite steady-state values. Note that $V_{5}$ is positive definite and radically unbounded. By LaSalle-Yoshizawa theorem [26], it follows that $\lim _{t \rightarrow \infty} W(\tilde{e})=0$, implying that $\tilde{e}(t) \rightarrow 0$, as $t \rightarrow \infty$, which further implies that $e(t) \rightarrow 0$, as $t \rightarrow \infty$. This completes the proof.

Remark 4. Theorem 5 shows that the adaptive consensus protocol (2) given by Theorem 1 is applicable to arbitrary switching communication graphs which are connected at any time instant. 
The case with switching leader-follower graphs can be discussed similarly, thus is omitted here for brevity. Because the Lyapunov function in (17) for the adaptive protocol (3) is explicitly related with the communication graph, it cannot be taken as a feasible common Lyapunov function.

\section{Simulation Examples}

In this section, a simulation example is provided to validate the effectiveness of the theoretical results.

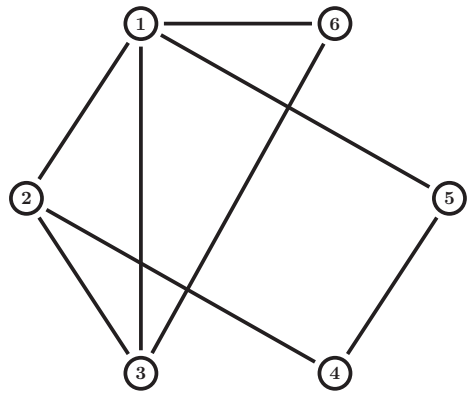

(a) $\mathcal{G}_{1}$

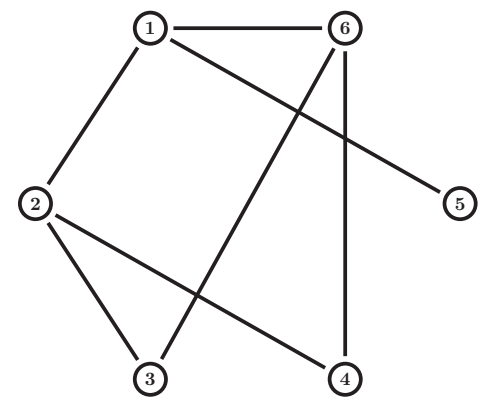

(b) $\mathcal{G}_{2}$

Figure 1: Undirected communication graphs $\mathcal{G}_{1}$ and $\mathcal{G}_{2}$.

Consider a network of third-order integrators, described by (11) with

$$
A=\left[\begin{array}{lll}
0 & 1 & 0 \\
0 & 0 & 1 \\
0 & 0 & 0
\end{array}\right], \quad B=\left[\begin{array}{l}
0 \\
0 \\
1
\end{array}\right], \quad C=\left[\begin{array}{lll}
1 & 0 & 0
\end{array}\right] .
$$

Choose $F=-\left[\begin{array}{lll}3 & 6.5 & 4.5\end{array}\right]$ such that $A+B F$ is Hurwitz. Solving the LMI (5) by using the LMI toolbox of Matlab gives the gain matrix $L$ in (33) and (3) as $L=-\left[\begin{array}{lll}2.5039 & 1.9056 & 0.9194\end{array}\right]^{T}$. To illustrate Theorem 5 , let $\mathcal{G}_{\sigma(t)}$ switch randomly every 0.1 second between $\mathcal{G}_{1}$ and $\mathcal{G}_{2}$ as shown in Figure 1. Note that both $\mathcal{G}_{1}$ and $\mathcal{G}_{2}$ are connected. Let $\kappa_{i j}=1, i, j=1, \cdots, 6$, in (33), and $c_{i j}(0)=c_{j i}(0)$ be randomly chosen. The consensus errors $x_{i}-x_{1}, i=2, \cdots, 5$, of the third-order integrators under the protocol (2) with $F, L$ as above and $\Gamma=\left[\begin{array}{cc}1 & -1 \\ -1 & 1\end{array}\right]$ are depicted in Figure 2. The coupling weights $c_{i j}$ associated with the edges in this case are shown in Figure 3. To illustrate Theorem 2, let the communication graph be $\mathcal{G}_{1}$ in Figure 1 (a) and $\tau_{i}=1, i=1, \cdots, 6$, in (3). The consensus errors $x_{i}-x_{1}, i=2, \cdots, 5$, of the third-order integrators under the protocol (3) with $F, L$, and $\Gamma$ as above are depicted in Figure 4. The coupling weights $d_{i}$ associated with the nodes are drawn in Figure 5. Figures 2 and 4 state that consensus is indeed achieved in both cases. From Figures 3 and 5, it can be observed that the coupling weights converge to finite steady-state values. 


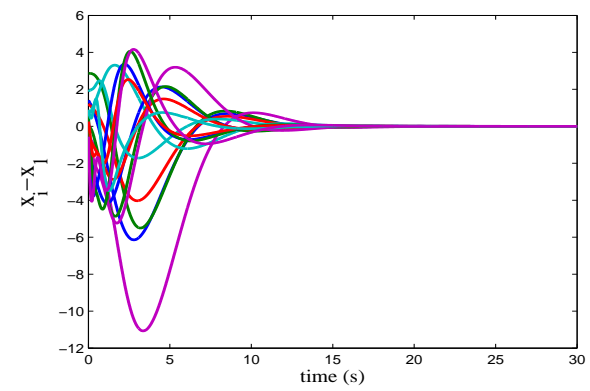

Figure 2: The consensus errors $x_{i}-x_{1}$ of third-order integrators under (33).

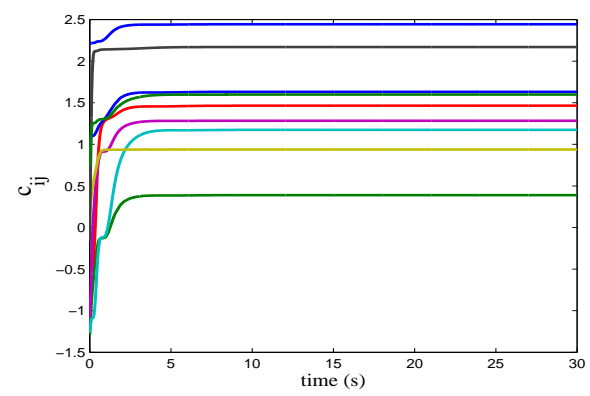

Figure 3: The coupling weights $c_{i j}$ associated with the edges in (33).

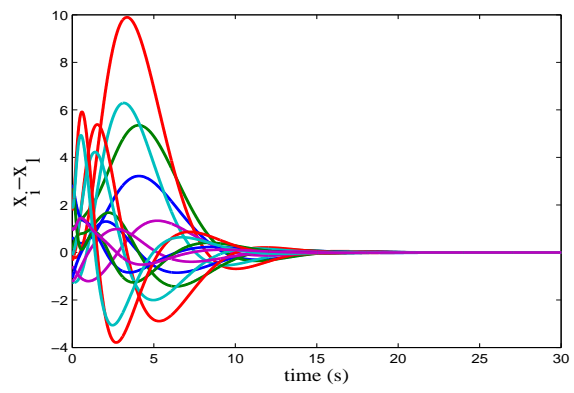

Figure 4: The consensus errors $x_{i}-x_{1}$ of third-order integrators under (3).

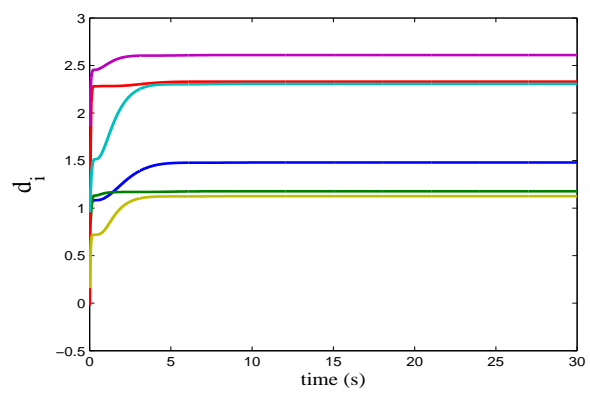

Figure 5: The coupling weights $d_{i}$ associated with the nodes in (3). 


\section{Conclusion}

In this paper, the consensus problem of multi-agent systems with identical general linear dynamics has been considered. Based on the relative output information of neighboring agents, two distributed adaptive dynamic consensus protocols have been proposed, namely, one protocol assigns an adaptive coupling weight to each edge in the communication graph while the other uses an adaptive coupling weight for each node. These two adaptive protocols have been designed to ensure that consensus is reached in a fully distributed fashion for any undirected connected communication graphs without using any global information.

\section{References}

[1] A. Jadbabaie, J. Lin, and A. Morse, "Coordination of groups of mobile autonomous agents using nearest neighbor rules," IEEE Transactions on Automatic Control, vol. 48, no. 6, pp. 988-1001, 2003.

[2] R. Olfati-Saber and R. Murray, "Consensus problems in networks of agents with switching topology and time-delays," IEEE Transactions on Automatic Control, vol. 49, no. 9, pp. 1520-1533, 2004.

[3] W. Ren and R. Beard, "Consensus seeking in multiagent systems under dynamically changing interaction topologies," IEEE Transactions on Automatic Control, vol. 50, no. 5, pp. 655-661, 2005.

[4] R. Olfati-Saber, J. Fax, and R. Murray, "Consensus and cooperation in networked multi-agent systems," Proceedings of the IEEE, vol. 95, no. 1, pp. 215-233, 2007.

[5] W. Ren, R. Beard, and E. Atkins, "Information consensus in multivehicle cooperative control," IEEE Control Systems Magazine, vol. 27, no. 2, pp. 71-82, 2007.

[6] W. Ren, "On consensus algorithms for double-integrator dynamics," IEEE Transactions on Automatic Control, vol. 53, no. 6, pp. 1503-1509, 2008.

[7] T. Li, M. Fu, L. Xie, and J. Zhang, "Distributed consensus with limited communication data rate," IEEE Transactions on Automatic Control, vol. 56, no. 2, pp. 279-292, 2011.

[8] A. Rahmani, M. Ji, M. Mesbahi, and M. Egerstedt, "Controllability of multi-agent systems from a graph-theoretic perspective," SIAM Journal on Control and Optimization, vol. 48, no. 1, pp. 162186, 2009.

[9] Y. Hong, G. Chen, and L. Bushnell, "Distributed observers design for leader-following control of multi-agent networks," Automatica, vol. 44, no. 3, pp. 846-850, 2008.

[10] J. Hu and G. Feng, "Distributed tracking control of leader-follower multi-agent systems under noisy measurement," Automatica, vol. 46, no. 8, pp. 1382-1387, 2010.

[11] W. Ren, K. Moore, and Y. Chen, "High-order and model reference consensus algorithms in cooperative control of multivehicle systems," ASME Journal of Dynamic Systems, Measurement, and Control, vol. 129, no. 5, pp. 678-688, 2007.

[12] F. Jiang and L. Wang, "Consensus seeking of high-order dynamic multi-agent systems with fixed and switching topologies," International Journal of Control, vol. 85, no. 2, pp. 404-420, 2010. 
[13] R. Carli, F. Bullo, and S. Zampieri, "Quantized average consensus via dynamic coding/decoding schemes," International Journal of Robust and Nonlinear Control, vol. 20, no. 2, pp. 156-175, 2009.

[14] Z. Li, Z. Duan, G. Chen, and L. Huang, "Consensus of multiagent systems and synchronization of complex networks: A unified viewpoint," IEEE Transactions on Circuits and Systems I: Regular Papers, vol. 57, no. 1, pp. 213-224, 2010.

[15] Z. Li, X. Liu, P. Lin, and W. Ren, "Consensus of linear multi-agent systems with reduced-order observer-based protocols," Systems $\&$ Control Letters, vol. 60, no. 7, pp. 510-516, 2011.

[16] S. Tuna, "Conditions for synchronizability in arrays of coupled linear systems," IEEE Transactions on Automatic Control, vol. 54, no. 10, pp. 2416-2420, 2009.

[17] J. Seo, H. Shim, and J. Back, "Consensus of high-order linear systems using dynamic output feedback compensator: Low gain approach," Automatica, vol. 45, no. 11, pp. 2659-2664, 2009.

[18] L. Scardovi and R. Sepulchre, "Synchronization in networks of identical linear systems," Automatica, vol. 45, no. 11, pp. 2557-2562, 2009.

[19] H. Zhang, F. Lewis, and A. Das, "Optimal design for synchronization of cooperative systems: State feedback, observer, and output feedback," IEEE Transactions on Automatic Control, vol. 56, no. 8, pp. 1948-1952, 2011.

[20] C. Ma and J. Zhang, "Necessary and sufficient conditions for consensusability of linear multi-sgent systems," IEEE Transactions on Automatic Control, vol. 55, no. 5, pp. 1263-1268, 2010.

[21] Z. Li, W. Ren, X. Liu, and M. Fu, "Consensus of multi-agent systems with general linear and Lipschitz nonlinear dynamics using distributed adaptive protocols," IEEE Transactions on Automatic Control, revised, 2011.

[22] P. De Lellis, M. di Bernardo, and F. Garofalo, "Synchronization of complex networks through local adaptive coupling," Chaos, vol. 18, p. 037110, 2008.

[23] W. Yu, W. Ren, W. Zheng, G. Chen, and J. Lv, "Distributed control gains design for consensus in multi-agent systems with second-order nonlinear dynamics," submitted to Automatica, 2011.

[24] C. Godsil and G. Royle, Algebraic graph theory. New York, NY: Springer-Verlag, 2001.

[25] S. Boyd, L. El Ghaoui, E. Feron, and V. Balakrishnan, Linear Matrix Inequalities in System and Control Theory. Philadelphia, PA: SIAM, 1994.

[26] M. Krstić, I. Kanellakopoulos, and P. Kokotovic, Nonlinear and Adaptive Control Design. New York: John Wiley \& Sons, 1995. 